\title{
The trans-Neptunian object (42355) Typhon: composition and dynamical evolution ${ }^{\star}$
}

\author{
A. Alvarez-Candal ${ }^{1,2}$, M. A. Barucci ${ }^{1}$, F. Merlin ${ }^{3}$, C. de Bergh ${ }^{1}$, S. Fornasier ${ }^{1}$, A. Guilbert ${ }^{1}$, and S. Protopapa ${ }^{4}$ \\ 1 LESIA/Observatoire de Paris, 5 place Jules Janssen, 92195 Meudon Cedex, France \\ 2 European Southern Observatory, Alonso de Córdova 3107, Vitacura Casilla 19001, Santiago 19, Chile \\ e-mail: aalvarez@eso.org \\ 3 Department of Astronomy, University of Maryland, College Park, MD 20742, USA \\ 4 Max-Planck Institute for Solar System Research, Max-Planck-Str. 2, 37191 Katlenburg-Lindau, Germany
}

Received 10 August 2009 / Accepted 17 November 2009

\section{ABSTRACT}

\begin{abstract}
Context. The scattered disk object (42355) Typhon shows interesting features in visible and near-infrared spectra, in particular, the visible spectrum shows evidence of aqueously altered materials.

Aims. This article presents a possible origin for absorption features on the surface of (42355) Typhon based on an episode of aqueous alteration, and it seeks to understand this event in the context of its dynamical evolution.

Methods. We observed (42355) Typhon at the ESO/Very Large Telescope using FORS2 and ISAAC on telescope unit 1 and SINFONI on telescope unit 4 . We compared these data with those previously published, in order to confirm features found in the visible and near infrared spectra and to study possible surface heterogeneities. We interpreted the surface composition using the Hapke radiative transfer model on the whole available spectral range $\sim 0.5-2.4 \mu \mathrm{m}$. To complete the portrait of (42355) Typhon, we followed its dynamical evolution using the code EVORB v.13 for 20 Myr.

Results. We confirm detection of a subtle absorption feature in the visible at $\sim 0.6 \mu \mathrm{m}$, which we interpret as caused by water-altered silicates. In the near infrared, we confirm the presence of water ice by the $2.0 \mu \mathrm{m}$ absorption feature. The best-fit models to our data point the presence of water ice.

Conclusions. (42355) Typhon is too small to have suffered water alteration, but this event could happen in a larger parent body from which (42355) Typhon is a remnant after a catastrophic disruption.
\end{abstract}

Key words. techniques: spectroscopic - Kuiper belt objects: individual: (42355) Typhon

\section{Introduction}

Trans-Neptunian objects (TNOs) are the remains of the building blocks of planets and the most primitive objects in the Solar System. Therefore, studying them gives insight into the conditions during the formation of the Solar System, and into how it was shaped and has evolved until the present day.

Aiming at obtaining high-quality data of the TNO population, a large program for the observation of centaurs and TNOs was started using the facilities of the ESO/Very Large Telescope at Cerro Paranal in Chile (PI: M.A. Barucci). The program lasted from November 2006 until December 2008. One of the main objectives was to increase the current database and quality of TNO spectra, thus increasing our statistical knowledge of the population. In the framework of the large program we were also able to identify interesting objects for further detailed individual studies.

One such object is (42355) Typhon (provisional designation $2002 \mathrm{CR}_{46}$ ). It is a scattered disk object (SDO), according to the classification scheme of Gladman (2008), and its perihelion distance (17.5 AU) lies inside the orbit of the icy giant planets. It was spectroscopically observed for the first time during the first year of the large program (2007 January) by AlvarezCandal et al. (2008) in the visible and Guilbert et al. (2009a) in

* Based on observations made with ESO Telescopes at the Paranal Observatories under programme ID 178.C-0036. the near infrared. While many TNOs do not show any spectral signature of ices in their spectra, we found in the visible spectrum of (42355) Typhon a subtle absorption feature (AlvarezCandal et al. 2008, AC08 hereafter) that could possibly be explained by aqueously altered minerals on its surface such as oxide hematite, oxyhydroxide goethite, or sulfate jarosite (see Vilas et al. 1994). In addition, (42355) Typhon, which belongs to the BR taxonomic class (DeMeo et al. 2009), is also covered by some water ice (Guilbert et al. 2009a).

(42355) Typhon was discovered to be the primary body of a binary system by Noll et al. (2006), with the secondary called Echidna. Because of the high normalized angular momentum, $J / J^{\prime}=2.13$, of the system, the most probable mechanism for its origin, as well as for most of the TNO binaries, is gravitational capture (Noll et al. 2008). The albedo of (42355) Typhon, based on its thermal emission, is $5.1_{-0.9}^{+1.3 \%}$, which corresponds to a diameter of about $175 \mathrm{~km}$ (Stansberry et al. 2008); however, this measurement could be overestimated due to the binary nature of (42355) Typhon. The magnitude contrast between the two bodies is $\Delta m=1.47$, which corresponds to

$\frac{\text { flux }_{\text {Echidna }}}{\text { flux }_{\text {Typhon }}} \sim 0.25$.

Because of this contrast we assume in what follows that the measured flux comes from (42355) Typhon. Moreover, Benecchi et al. (2009) have recently shown that the colors of both bodies 
are similar, therefore the slope of the spectrum of the integrated system applies to any of the objects.

To confirm the previous detection of a weak band in the visible and to look for possible surface heterogeneities, we observed (42355) Typhon again, with the telescopes at the Paranal Observatory in Chile. We present a short description in Sect. 2 of the dynamical evolution of (42355) Typhon. The observations are presented in Sect. 3, while in Sect. 4 we compare the new data with those obtained one year earlier and present our results on the surface composition of (42355) Typhon based on a modeling of the complete visible-near infrared spectrum, using a Hapke radiative-transfer model. Finally, in Sect. 5 we discuss the obtained results.

\section{Dynamics of (42355) Typhon}

Scattered disk objects are known to have suffered close encounters with planets (Duncan \& Levison 1997), which could have affected their internal structure. Therefore, (42355) Typhon's physical/chemical evolution depends, among other factors, on its dynamical evolution, so we followed it by integrating its orbital elements over $2 \times 10^{7} \mathrm{yr}$ using an integration step of $5 \times 10^{-3} \mathrm{yr}$ and outputs each $50 \mathrm{yr}$ increments. The current orbital elements of (42355) Typhon are: $a=37.7 \mathrm{AU}, e=0.54$, and $i=2.43^{\circ}$, from the Lowell Observatory. $a, e$, and $i$ denote the usual osculating orbital elements.

We followed the evolution of a test particle with (42355) Typhon's orbital elements (for the epoch JD 2454707.5, as taken from the astorb.dat, ftp: //ftp. lowell.edu/pub/elgb/astorb.html data file). To evaluate the effect of the uncertainties in (42355) Typhon's elements, we cloned them randomly 20 times in the intervals $0.01 \mathrm{AU}$ for $a, 0.01$ for $e$, and $0.5^{\circ}$ for the angular elements, around (42355) Typhon original elements. We then followed the evolution of the 21 test particles using the code EVORB v.13, provided by T. Gallardo. The code is a second-order symplectic integrator based on the algorithm written by Wisdom \& Holman (1991). The code handles close encounters with planets via a Bulirsch-Stoer routine. For details about the accuracy of the code see, Fernández et al. (2002). The particles were integrated under the influence of the eight planets. We did not consider any non-gravitational force.

According to the Gladman classification scheme, (42355) Typhon is currently an SDO. Its perihelion distance, 17.3 AU, places it in an orbit that crosses orbits of the icy giants. Therefore, it is safe to mention that all 21 particles will have centaur-like orbits, which is corroborated by our integrations. Among the sample, nine test particles abandon the integration before the end, and six of them, (42355) Typhon included, reach Jupiter family comet (JFC) orbits, defined as $T_{\mathrm{J}}<3$, where $T_{\mathrm{J}}$ is the Tisserand parameter with respect to Jupiter.

Figure 1 shows the evolution of (42355) Typhon's semimajor axis $(a)$, perihelion $(q)$, and aphelion $(Q)$ distances. The test particle suffers large excursions in eccentricity and inclination, and it has close encounters with the giant planets. These excursions seem to be related, although not always. The same behavior is seen for other test particles. It alternatively encounters Uranus and Neptune until about $2.5 \times 10^{6} \mathrm{yr}$, when it has an encounter with Saturn that changes its semi-major axis from about 22 to $40 \mathrm{AU}$ ( $e$ jumps from 0.5 to 0.7 ), which eventually reaches values $\sim 50 \mathrm{AU}$. At $3.9 \times 10^{6} \mathrm{yr}$, the semi-major axis starts decreasing, while the eccentricity decreases from about 0.8 until 0.5 . Then the test particle begins suffering encounters with Jupiter, so that its orbit finally evolves into a JFC-like one.

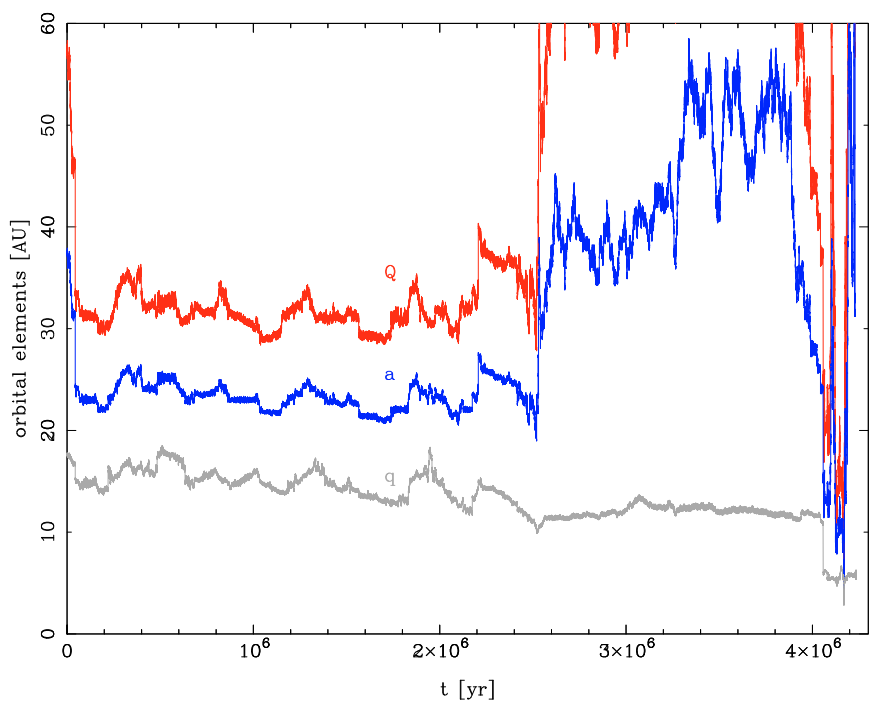

Fig. 1. Time evolution of a test particle with the initial elements of (42355) Typhon. The total integration time was $2 \times 10^{7}$ yr. The blue (middle) line indicates the evolution of the semi-major axis, $a$, while the red (upper) and gray (lower) ones indicate those of the aphelion, $Q$, and perihelion, $q$, distances, respectively. The distance between $a$ and $q$, or $Q$, shows the change in eccentricity. The largest excursion in eccentricity was $\sim 0.6$, while in inclination was $\sim 20^{\circ}$.

Shortly after, it leaves the integration after a close encounter with Jupiter. The inclination (not shown) suffers changes from near $0.5^{\circ}$ up to $21.5^{\circ}$. (42355) Typhon leaves the integration at about $4.5 \times 10^{6} \mathrm{yr}$ (reaching $\left.a>300 \mathrm{AU}\right)$.

\section{Observations and data reduction}

We observed (42355) Typhon during two runs at the Very Large Telescope/ESO in 2007 January and 2008 April, using two telescopes and three instruments. (42355) Typhon was observed with FORS2 and ISAAC at the UT 1 and SINFONI at the UT 4. Since FORS2 and ISAAC are both in the same unit, it was not possible to observe (42355) Typhon at all wavelengths simultaneously. Moreover, bad conditions made it impossible to observe (42355) Typhon with ISAAC during the 2008 April run.

Data from the first observing run in 2007 January have already been published: VRIJHK photometry by DeMeo et al. (2009), visible spectroscopy by AC08, and $H+K$ spectroscopy by Guilbert et al. (2009a). Therefore, in what follows, we concentrate on the $J$-spectrum obtained in the 2007 January run, previously unpublished and on the new photometric and spectroscopic data obtained during the 2008 April run. The observational circumstances are reported in Table 1.

\subsection{Photometry}

We used FORS2 at the UT 1 with the broadband Bessel filters VRI, centered at $0.554,0.657$, and $0.768 \mu \mathrm{m}$, respectively. The detector at FORS2 is optimized for the red and consists of a mosaic of two $2 k \times 4 k$ MIT CCDs (with $15 \mu \mathrm{m}$ pixels). The highgain mode (1.45 $\mathrm{e}^{-} /$ADU) was used for the read out of the CCD.

The data reduction was performed following standard techniques with IRAF. Daylight calibration files provided by the ESO calibration unit were used for bias-subtraction and flatfielding. The magnitude of (42355) Typhon was measured by aperture photometry, typically 3 times the FWHM. Standard stars were also observed and calibrated with the same setup as 
Table 1. Observational circumstances.

\begin{tabular}{lclcccl}
\hline \hline Instrument & Range $(\mu \mathrm{m})$ & Date & Exposure (s) & Airmass & Seeing & Analog Star (Airmass) \\
\hline FORS2 $^{a}$ & 0.554 & 12 Apr. 2008 & 10 & 1.25 & 0.8 & - \\
FORS2 $^{a}$ & 0.657 & 12 Apr. 2008 & 10 & 1.24 & 0.8 & - \\
FORS2 $^{a}$ & 0.768 & 12 Apr. 2008 & 20 & 1.24 & 0.8 & - \\
FORS2 $^{b}$ & $0.4-0.9$ & 12 Apr. 2008 & 1200 & 1.22 & 0.8 & SA102-1081 (1.10) \\
ISAAC $^{b}$ & $1.15-1.35$ & 24 Jan. 2007 & 180 & 1.22 & 0.6 & SA102-1081 (1.14) \\
SINFONI $^{b}$ & $1.78-2.35$ & 12 Apr. 2008 & 7800 & 1.23 & 0.8 & SA102-1081 (1.23) \\
\hline
\end{tabular}

Notes. ${ }^{(a)}$ Photometry . ${ }^{(b)}$ Spectroscopy .

Table 2. Photometric results of (42355) Typhon

\begin{tabular}{lcccccc}
\hline \hline Ref. & $V$ & $(V-R)$ & $(V-I)$ & $(V-J)$ & $(V-H)$ & $(V-K)$ \\
\hline$a$ & $20.50 \pm 0.08$ & $0.50 \pm 0.10$ & $0.80 \pm 0.10$ & & & \\
$b$ & & $0.55 \pm 0.05$ & $0.99 \pm 0.06$ & $1.83 \pm 0.09$ & $2.18 \pm 0.12$ & $2.34 \pm 0.13$ \\
$c$ & $20.34 \pm 0.02$ & $0.48 \pm 0.04$ & $0.94 \pm 0.03$ & $1.56 \pm 0.04$ & $2.01 \pm 0.05$ & $2.17 \pm 0.05$ \\
$d$ & & & $0.96 \pm 0.02$ & & & \\
\hline
\end{tabular}

Notes. $a$ - This work. $b$ - Doressoundiram et al. (2007). $c$ - DeMeo et al. (2009). $d$ - Benecchi et al. (2009), value only for the primary body.

the object. Extinction was corrected with the mean coefficients provided by ESO for the Paranal observatory. The standard magnitude of (42355) Typhon was computed using the zero point determined from Landolt (1992) standard stars observed during the night.

Due to bad weather conditions during the second night of the run, we were not able to obtain any near-infrared photometric data of (42355) Typhon.

\subsection{Spectroscopy}

\subsubsection{FORS2}

The observations were obtained with the FORS2 instrument using a low-resolution grism (150 grooves $/ \mathrm{mm})$ with a $1^{\prime \prime}$ wide slit, covering the $0.4-0.9 \mu \mathrm{m}$ wavelength range with a spectral resolution of about 200 . The slit was oriented along the parallactic angle to minimize the effects of atmospheric differential refraction, which is also instrumentally corrected by a system of two silica prisms.

The data was reduced using IRAF. For the bias correction, flat-fielding, and wavelength calibration (HeAr lamps) daylight calibration files were used. After extraction, (42355) Typhon spectra were divided by that of a solar analog star observed with the same setup as the object and matching the airmass as much as possible. The spectra were then cleaned using a median filter, as described in AC08.

\subsubsection{ISAAC}

We used ISAAC in 2007 January to obtain the J spectroscopic region from 1.15 to $1.35 \mu \mathrm{m}$. We used the SW mode of the instrument with a $1^{\prime \prime}$ slit giving a spectral resolution of about 500 . The observations were done by nodding the object along the slit by $10^{\prime \prime}$ between two positions. Each pair of images was combined using the MIDAS software package following the procedures described in Barucci et al. (2002). The wavelength was calibrated using Xenon and Argon lamp spectral lines. The reflectivity of the object was obtained by dividing its spectrum by that of a solar analog star observed at similar airmass.

\subsubsection{SINFONI}

We used the $H+K$ grating of SINFONI to obtain (42355) Typhon's spectrum. This grating covers the $H$ and $K$ bands simultaneously with a resolution of about 1500 and a plate scale of $0.25^{\prime \prime} /$ pixel. The data were reduced using the SINFONI pipeline v.1.8 ${ }^{1}$. The total exposure time was split into 13 images of 10 min each taken following a dithering pattern to have a good sampling of the sky. The single images were combined to obtain the final science cube.

The spectra were extracted from the individual cubes using QFitsView, a 3D visualization tool developed at the Max Planck Institut für Extraterrestrische Physik. (42355) Typhon's spectrum was then divided by the solar analog spectrum to remove the solar contribution, as well as telluric features. Finally, the spectrum was corrected for any remaining bad pixels. The wavelength calibration was performed using xenon, argon, and krypton lamps.

More detailed information about the handling of SINFONI data of TNOs can be found in Guilbert et al. (2009a).

\section{Results}

The new photometric data and colors are reported in Table 2. We also included the colors measured by Doressoundiram et al. (2007), DeMeo et al. (2009), and Benecchi et al. (2009). From the table we see that the visible colors all agree at the 2- $\sigma$ level, even if our $(V-I)$ color is a bit less than previously published ones. The errors in April's colors are larger than the others, which may be due to poorer atmospheric conditions.

Figure 2 shows the two composite spectra of (42355) Typhon from 0.4 to $2.3 \mu \mathrm{m}$. The spectrum presented in this work is labeled Apr08, while the composite spectrum of the data already published is labeled Jan07. In this last case, the $J$-spectrum taken with ISAAC, which has not been previously published, is also included. To connect the entire spectral range, we used the colors $(V-J)$ and $(V-H)$ from DeMeo et al. (2009). The $(V-K)$ color is in good agreement with the overall spectral behavior.

Our visible spectrum is similar to that of AC08, and the signal-to-noise ratio decreased from 68@0.55 $\mu \mathrm{m}$ in the 2007

\footnotetext{
1 ftp://ftp.eso.org/pub/dfs/pipelines/sinfoni/ sinfo-pipeline-manual-10.0.pdf
} 


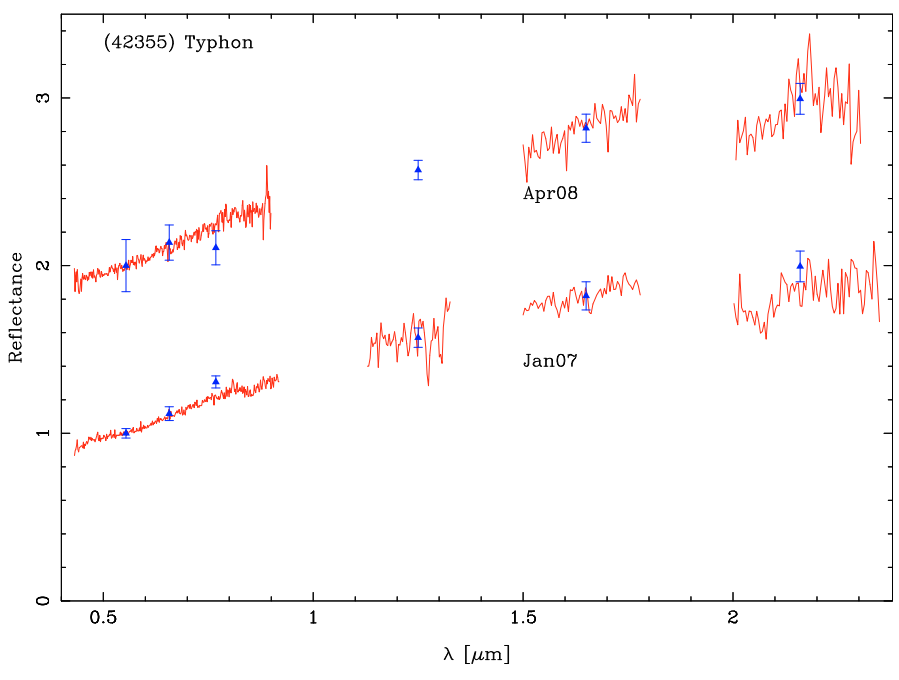

Fig. 2. Composite spectrum of (42355) Typhon obtained with the data taken in the 2007 January and 2008 April runs. The spectra are normalized to unity at the $V$-filter, and the 2008 April spectrum is shifted by 1 for clarity. The blue triangles show the photometric colors used to set the reflectance scale. The region between 1.8 and $2.0 \mu \mathrm{m}$ was removed due to the strong telluric absorptions.

January spectrum to $37 @ 0.55 \mu \mathrm{m}$ in 2008 April because of poorer atmospheric conditions and a shorter exposure time (Fig. 2). Curiously, the $(V-R)$ color in both observations matches the spectra at a $1-\sigma$ level, while for the $(V-I)$ color is at a 2- $\sigma$ level. The spectral gradient, $S^{\prime 2}$ are similar in the visible of both spectra $10.6 \pm 0.6$ and $11.7 \pm 0.5 \%(0.1 \mu \mathrm{m})^{-1}$, for the 2007 January and 2008 April observations, respectively. Our SINFONI spectrum closely resembles that of Guilbert et al. (2009a). The similar behavior of the spectra suggests homogeneity of the surface composition.

\subsection{Features}

In AC08 we suggested that (42355) Typhon, together with (10199) Chariklo and $2003 \mathrm{AZ}_{84}$, show evidence of a shallow broad band in the visible spectrum. We proposed that it could stem from the presence of aqueously altered minerals on (42355) Typhon's surface, as previously proposed for (38628) Huya and (47932) $2000 \mathrm{GN}_{171}$ by Lazzarin et al. (2003). In this last case the detection was never confirmed (de Bergh et al. 2004; Fornasier et al. 2004).

We processed both visible spectra, that of 2007 January and that of 2008 April, by adapting the technique described in Vilas et al. (1993, 1994) and Fornasier et al. (1999). We smoothed the spectra using a window of about $0.03 \mu \mathrm{m}$, and then we estimated the slope of the continuum, tracing a line between 0.47 and $0.78 \mu \mathrm{m}$ (e.g., Carvano et al. 2003). The spectra were then divided by this conventional continuum. These "normalized" spectra are shown in Fig. 3. There is evidence of an absorption feature in the 2008 April spectrum, even deeper than observed in 2007 January although the shape and position are slightly different (see Table 3).

The band depth of the spectra was measured by computing the value of the normalized reflectance at $\lambda_{\mathrm{c}}$ :

$D[\%]=100 \times\left(1-\right.$ flux $\left._{\lambda_{\mathrm{c}}}\right)$.

\footnotetext{
${ }^{2}$ Computed between 0.5 and $0.8 \mu \mathrm{m}$.
}

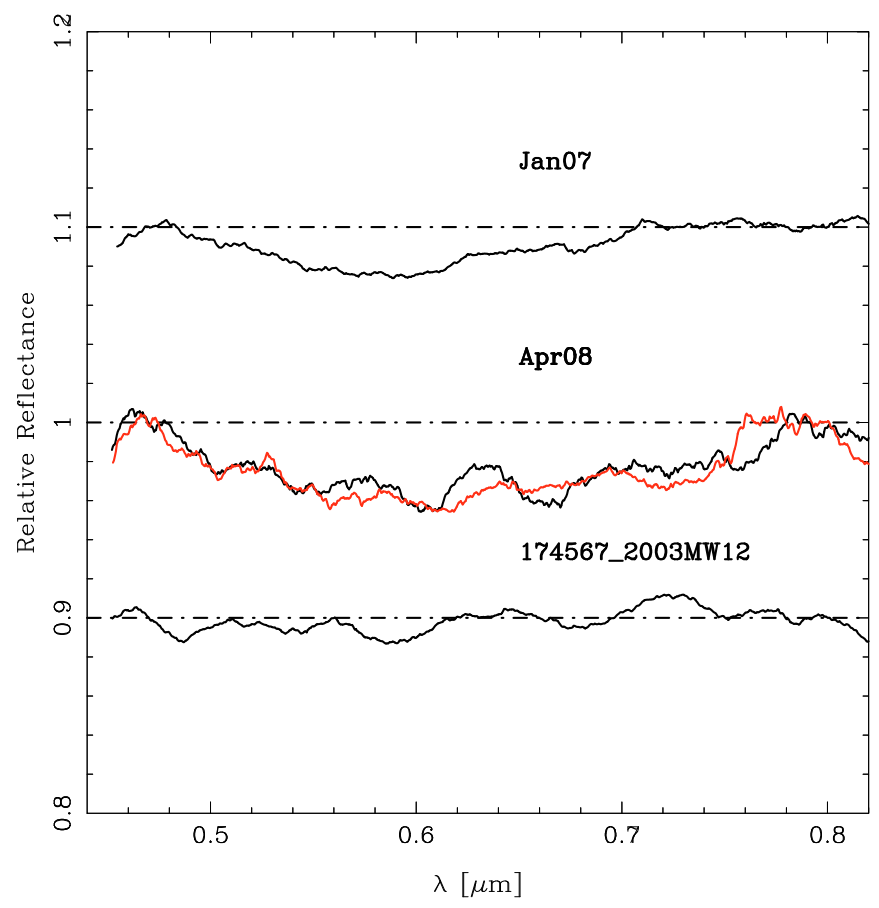

Fig. 3. Visible spectra of (42355) Typhon after extraction of the continuum. The spectrum labeled Apr08 is presented in this work, while the spectrum from the 2007 January run (AC08) is offset by a reflectance of 0.1 for comparison. In red we show the spectrum of (42355) Typhon as obtained dividing by a different solar analog observed the same night. To illustrate that the absorption we see not comes from our choice of calibration star, we apply the same analysis to the spectra of (174567) $2003 \mathrm{MW}_{12}$, obtained the same night as our (43355) Typhon data, and find no evidence of absorption.

Because of the noise in the spectra and the subsequent difficulty in defining the band limits, the numerical values listed in Table 3 are only indicative.

The feature is still present when dividing by other solar analog stars observed the same night (red colored spectrum overplotted). To rule out possible problems with the observations, we checked other objects observed the same night, with the same observational setup and did not detect similar features (2003 $\mathrm{MW}_{12}$ in Fig. 3).

A band centered at about $0.6 \mu \mathrm{m}$ due to aqueously alteration is usually accompanied by a second band centered at $\sim 0.9 \mu \mathrm{m}$. Unfortunately, the FORS spectra show an important decrease in the signal-to-noise ratio above $0.8 \mu \mathrm{m}$.

We only have one $J$-spectrum recorded for (42355) Typhon, which is noisy and apparently featureless. Concerning the $H+K$ spectra, we detect the presence of water ice, mostly from the absorption at $2 \mu \mathrm{m}$. The counterpart at $1.5 \mu \mathrm{m}$ is less evident because of the low signal-to-noise of the spectrum. We computed the depth of the $2.0 \mu \mathrm{m}$-band of water ice for both spectra, defined as

$D[\%]=100 \times\left(1-\frac{\text { flux }_{1.7 \mu \mathrm{m}}}{\text { flux }_{2.0 \mu \mathrm{m}}}\right)$,

obtaining values of $10 \pm 4 \%$ and $14 \pm 7 \%$, for the 2007 January and the 2008 April spectra, respectively. The apparent feature in the 2007 January spectrum at $2.25 \mu \mathrm{m}$ is probably an artifact of the combination of decreasing efficiency of the detector and the faintness of the target. 
Table 3. Spectroscopic results of (42355) Typhon.

\begin{tabular}{lccccc}
\hline \hline Ref. & Range & Feature? & $\lambda_{\mathrm{c}}[\mu \mathrm{m}]$ & ${\text { Depth }(\%)^{a}}^{a}$ & Interpretation \\
\hline$b$ & Vis. & yes & 0.62 & $3 \pm 1$ & Aqueous Alteration? \\
$c$ & Vis. & yes & 0.58 & $1 \pm 1$ & Aqueous Alteration? \\
$b$ & $J$ & no & - & - & - \\
$b$ & $H+K$ & yes & 2.0 & $14 \pm 7$ & $\mathrm{H}_{2} \mathrm{O}$ \\
$d$ & $H+K$ & yes & 2.0 & $10 \pm 4$ & $\mathrm{H}_{2} \mathrm{O}$ \\
\hline
\end{tabular}

Notes. $a$-Visible, computed following Eq. (1). Near infrared, computed following Eq. (2). $b$ - This work. $c$ - AC08. $d$ - Guilbert et al. (2009a).

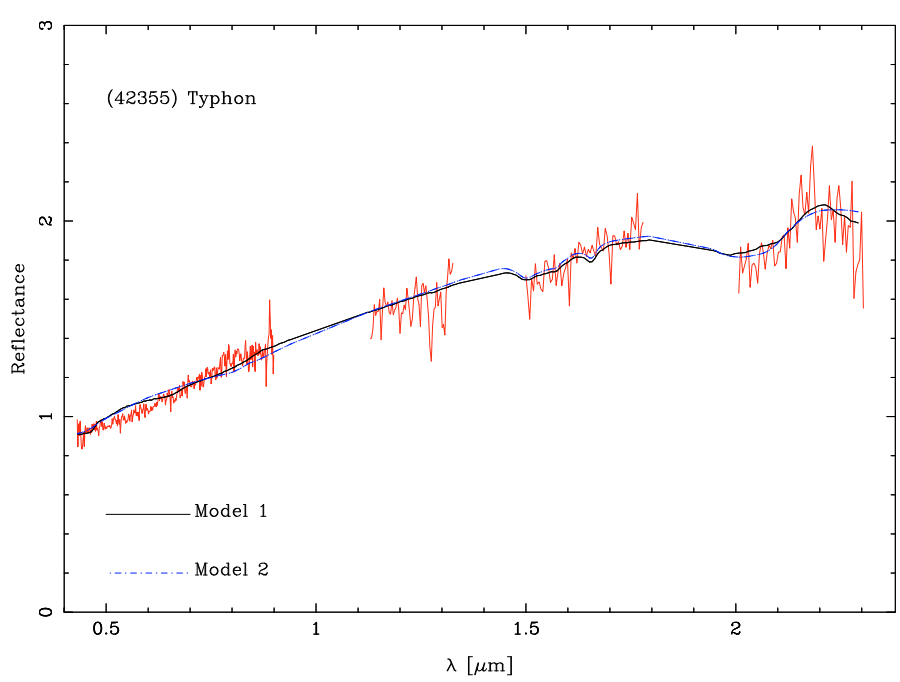

Fig. 4. Composite spectra of (42355) Typhon taken in 2008 April. The spectrum is normalized to unity at the $V$-filter. The black continuous line shows Model 1, while the blue dot-dashed line shows Model 2. Both models are described in Sect. 4.2.

\subsection{Modeling}

To investigate the surface properties of (42355) Typhon, we modeled the spectrum from the data obtained in 2008 April. We ran a radiative transfer model, based on the Hapke theory (Hapke 1981, 1993). The free parameters of the model are the compound grain size and abundance. The best-fit model was obtained by minimizing $\chi^{2}$ using a Marqvardt-Levenberg algorithm.

To model the spectral behavior and the detected signatures, we consider several components in our model. As the visible part of the spectrum has a red continuum, about $12 \%(0.1 \mu \mathrm{m})^{-1}$, we included in the model Triton and Titan tholins with optical constants from Khare et al. (1984, 1993). Because of the absorption signature of water ice at $2.0 \mu \mathrm{m}$, the model also includes water ice in crystalline and amorphous states (crystalline water ice constants at 40 K from Quirico \& Schmitt 1997; amorphous ice at $38 \mathrm{~K}$ from Grundy \& Schmitt 1998). To match the albedo of 5\%, we used amorphous carbon (Zubko et al. 1996). To account for the possible presence of aqueously altered minerals, we introduced serpentine in one of our models, as in Protopapa et al. (2009), which is the only hydrated mineral for which optical constants are available and which has a weak absorption in the visible range and an acceptable behavior in the near infrared. The optical constants for serpentine have been computed by R. Clark between $0.3-2.5 \mu \mathrm{m}$ (private communication).

Figure 4 shows the two best-fitting models, obtained using intimate mixtures: Model 1, corresponding to a model including $12 \%$ Serpentine $(160 \mu \mathrm{m}), 4 \%$ of crystalline water ice $(85 \mu \mathrm{m})$, 17 , and $6 \%$ of Triton and Titan tholins, respectively, and $61 \%$ of amorphous carbon. This model corresponds to an albedo of
$6.38 \%$ at $0.55 \mu \mathrm{m}$; and Model 2, including $6 \%$ of crystalline water ice $(45 \mu \mathrm{m}), 28 \%$ of Triton tholin $(13 \mu \mathrm{m}), 59 \%$ of Titan tholin $(1 \mathrm{~mm}), 6 \%$ of amorphous water ice $(10 \mu \mathrm{m})$, and $1 \%$ of amorphous carbon. The albedo at $0.55 \mu \mathrm{m}$ is $6.04 \%$. Both models give the same $\chi^{2}=0.05$, both models describe the whole spectral range reasonably well, although none of them is able to reproduce the feature detected in the visible.

\section{Discussion}

The new spectra of (42355) Typhon that we obtained at the UTs 1 and 4 of the Very Large Telescope in the framework of an ESO Large Program confirm the detection by Alvarez-Candal et al. (2008) of an absorption band in the visible at about $0.6 \mu \mathrm{m}$ and of the water ice band in the near infrared, around $2.0 \mu \mathrm{m}$, as reported by Guilbert et al. (2009a). In both cases, the depth of the features are comparable to those previously observed (see Table 3). Even if we do not have good constraint for the rotation period of (42355) Typhon (see Dotto et al. 2008), this favors of a homogeneous surface.

The feature detected at about $0.6 \mu \mathrm{m}$ resembles those found in some low-albedo asteroids (Vilas et al. 1994; Rivkin et al. 2002), which are often, but not uniquely (King \& Clark, 1997), associated to phyllosilicates, formed by the interaction of liquid water and silicates, and could indicate the presence of material that suffered aqueous alteration. The residuals of the telluric bands and the noise in the spectra may be responsible for the difference in the central wavelengths (Table 3) for the 2007 January and 2008 April runs, caused by the difficulty determining where the feature begins and ends.

Phyllosilicates could also have bands in the near infrared: $1.4,1.9,2.2-2.4$, and $3.0 \mu \mathrm{m}$. The only ones covered by our observations are those between 2.2 and $2.4 \mu \mathrm{m}$. Unfortunately, our spectra do not have enough signal-to-noise ratio to confidently confirm or reject any detection of such features.

Several potential mechanisms that could produce this alteration were discussed in de Bergh et al. (2004). We concentrate on the most plausible one: heating by decay of radiogenic materials, such as ${ }^{26} \mathrm{Al}$ (Urey 1955). It is known (see McKinnon et al. 2008, and references therein) that the interior of icy bodies could have undergone melting of the water ice via radiogenic heating. This is supported by the detection of hydrated silicates on comet nuclei (Lisse et al. 2006). Nevertheless, Prialnik et al. (2008) stress that it is more probable for the biggest objects, since melting requires high temperatures $(273 \mathrm{~K})$ and also high pressures (600 Pa).

Interestingly, Guilbert et al. (2009b) present new data of the centaur (10199) Chariklo showing a feature in the visible, probably indicating water alteration, similar to that of (42355) Typhon. (10199) Chariklo is in a centaur orbit, i.e. crosses the orbits of the giant planets. In Sect. 2 we showed that an SDO can become a centaur, even a JFC. Therefore (10199) Chariklo could have been an SDO in the past (Gomes et al. 2008). (10199) Chariklo 
is twice as big as (42355) Typhon, so it is possible that it had enough internal heat to melt water ice, which is unlikely to be the case of (42355) Typhon, which is too small to have suffered complete melting. The other TNOs or centaurs for which water alteration has been hypothesized (Sect. 4.1) have diameters in the $200-700 \mathrm{~km}$ range.

One possibility for explaining the water alteration on (42355) Typhon's surface is that a larger parent body, from which (42355) Typhon is only one of the remnants of a catastrophic collision, was large enough as to suffer melting in its interior, and this possibility is sustained by the existence of (10199) Chariklo's feature. Collisions among SDOs are hypothesized, for instance, to explain the dynamical group of (136108) Haumea (see Levison et al. 2008). Also, (42355) Typhon is a peculiar object on an unstable orbit (Sect. 2). It could become a centaur, and, eventually a Jupiter-family comet. Given its low perihelion distance, this object has most probably been subjected to several close encounters with planets in the past. These encounters could have affected its internal structure because of tidal forces, maybe even scrambling a possible rubble-pile structure, bringing buried altered material up to the surface and making it possible to detect materials that would otherwise remain hidden.

Acknowledgements. A.A.C. would like to thank the European Space Agency for financial support through the international traineeships program. We thank P. S. Lykawka for his comments about the dynamical evolution of (42355) Typhon, and M. Moerchen for revising the English of the manuscript. We also acknowledge the referee for his/her suggestions that helped to improve this manuscript.

\section{References}

Alvarez-Candal, A., Fornasier, S., Barucci, M. A., de Bergh, C., \& Merlin, F. 2008, A\&A, 487, 741

Barucci, M. A., Boehnhardt, H., Dotto, E., et al. 2002, A\&A, 392, 335

Benecchi, S. D., Noll, K. S., Grundy, W. M., et al. 2009, Icarus, 200, 292

Carvano, J. M., Mothé-Diniz, T., \& Lazzaro, D. 2003, Icarus, 161, 356

de Bergh, C., Boehnhardt, H., Barucci, M. A., et al. 2004, A\&A, 416, 791

DeMeo, F., Fornasier, S., Barucci, M. A., et al. 2009, A\&A, 493, 283

Doressoundiram, A., Peixinho, N., Moullet, A., et al. 2007, AJ, 134, 2186

Dotto, E., Perna, D., Barucci, M. A., et al. 2008, A\&A, 490, 829

Duncan, M. J., \& Levison, H. F. 1997, Science, 276, 1670

Fernández, J. A., Gallardo, T., \& Brunini, A. 2002, Icarus, 159, 358

Fornasier, S., Lazzarin, M., Barbieri, C., \& Barucci, M. A. 1999, A\&A, 135, 65
Fornasier, S., Doressoundiram, A., Tozzi, G. P., et al. 2004, A\&A, 421, 353

Gladman, B., Marsden, B. G., \& VanLaerhoven, C. 2008, Nomenclature in the outer Solar System, in The Solar System Beyond Neptune ed. M. A. Barucci, H. Boehnhardt, D. Cruikshank, \& A. Morbidelli (Tucson: Univ. of Arizona Press), 43

Gomes, R. S., Fernández, J. A., Gallardo, T., \& Brunini, A. 2008, The Scattered Disk: Origins, Dynamics, and End States, in The Solar System Beyond Neptune, ed. M. A. Barucci, H. Boehnhardt, D. Cruikshank, \& A. Morbidelli (Tucson: Univ. of Arizona Press), 259

Grundy, W. M., \& Schmitt, B. 1998, J. Geophys. Res., 103, 25809

Guilbert, A., Alvarez-Candal, A., Merlin, F., et al. 2009a, Icarus, 201, 272

Guilbert, A., Barucci, M. A., Brunetto, R., et al. 2009b, A\&A, 501, 777

Hapke, B. 1981, J. Geophys. Res., 86, 3039

Hapke, B. 1993, Topics in Remote Sensing 3: Theory of reflectance and emittance spectroscopy (Cambridge: Cambridge Univ. Press)

Khare, B. N., Sagan, C., Arakawa, E. T., et al. 1984, Icarus, 60, 127

Khare, B. N., Thompson, W. R., Cheng, L., et al. 1993, Icarus, 103, 290

King, T. V. V., \& Clark, R. N. 1997, Lun. Planet. Sci., XXVII, 727

Landolt, A. U. 1992, AJ, 104, 340, 436

Lazzarin, M., Barucci, M. A., Boehnhardt, H., et al. 2003, AJ, 125, 1554

Levison, H. F., Morbidelli, A., Vokrouhlický, D., \& Bottke, W. F. 2008, AJ, 136, 1079

Lisse, C. M., \& 16 colleagues 2006, Nature, 313, 635

McKinnon, W. B., Prialnik, D., Stern, S. A., \& Coradini, A. 2008, Structure and Evolution of Kuiper Belt Objects and Dwarf Planets, in The Solar System Beyond Neptune, ed. M. A. Barucci, H. Boehnhardt, D. Cruikshank, \& A. Morbidelli (Tucson: Univ. of Arizona Press), 213

Noll, K. S., Grundy, W. M., Stephens, D. C., \& Levison, H. F. 2006, IAU Circ., 8689,1

Noll, K. S., Grundy, W. M., Chiang, E. I., Margot, J.-L., \& Kern, S. D. 2008, Binaries in the Kuiper Belt, in The Solar System Beyond Neptune, ed. M. A. Barucci, H. Boehnhardt, D. Cruikshank, \& A. Morbidelli (Tucson: Univ. of Arizona Press), 345

Prialnik, D., Sarid, G., \& Rosenberg, E. D. 2008, Space Sci. Rev., 138, 147

Protopapa, S., Alvarez-Candal, A., Barucci, M. A., et al. 2009, A\&A, 501, 375

Quirico, E., \& Schmitt, B. 1997, Icarus, 127, 354

Rivkin, A. S., Howell, E. S., Vilas, F., \& Lebofsky, L. A. 2003, Hydrated Minerals on Asteroids: The Astronomical Record, in Asteroids III, ed. W. F. Bottke, A. Cellino, P. Paolicchi, \& R. P. Binzel (Tucson: Univ. of Arizona Press), 235

Stansberry, J., Grundy, W., Brown, M., et al. 2008, Physical Properties of Kuiper Belt and Centaur Objects: Constraints form the Spitzer Space Telescope, in The Solar System Beyond Neptune, ed. M. A. Barucci, H. Boehnhardt, D. Cruikshank, \& A. Morbidelli (Tucson: Univ. of Arizona Press), 161

Urey, H. C. 1955, Proc. Natl. Acad. Sci., 41, 127

Vilas, F., Hatch, E. C., Larson, S. M., Sawyer, S. R., \& Gaffey, M. J. 1993, Icarus, 102,225

Vilas, F., Jarvis, K. S., \& Gaffey, M. J. 1994, Icarus, 109, 274

Wisdom, J., \& Holman, M. 1991, AJ, 102, 1528

Zubko, V. G., Mennella, V., Colangeli, L., \& Bussoletti, E. 1996, MNRAS, 282, 1321 